\title{
Las declaraciones no son suficientes: las fuentes del sector financiero para la independencia del banco central
}

\section{Introducción}

Aun antes de la tan mencionada reestructuración del Banco de Reserva de Nueva Zelandia en 1990, a los bancos centrales se les han garantizado diferentes grados de independencia con respecto al control político a corto plazo. La discusión subyacente en esta moda actual sobre la independencia del banco central, ha supuesto que las opciones anteriores relacionadas con los regímenes monetarios eran aleatonias, en vez de interpretar la correlación negativa entre las tasas de inflación promedio y los índices de independencia del banco central, como una evidencia de prima facie de una relación causal o recomendando la independencia del banco central a cualquiera de los gobiernos, ya sea en París, Moscú o Karachi. En este artículo se sostiene que dicho supuesto de exogeneidad de las instituciones monetarias es falso. Las diferencias nacionales en el grado de independencia del banco central en el período posguerra se debieron, en cambio, a diferencias en la oposición de los sectores financieros a la inflación.

Todo en economía puede ser visto a cierto nivel como endógeno. Lo que hace que la cuestión de la endogeneidad de la independencia del banco central tenga significado, es apartarse de lo que uno esperaría si los modelos de inconsistencia en el tiempo ${ }^{2}$, normalmente invocados para explicar la correlación negativa entre la independencia del banco central (de aquí en adelante IBC) y la inflación, captaran adecuadamente el mundo de la política monetaria. Lo más importante es que la IBC no parece ser una mejora de Pareto (inclusive si en equilibrio mejora el bienestar). El costo de la desinflación medido en función del desempleo no solamente no deja de disminuir en un régimen monetario más creíble de mayor IBC, sino que parece aumentar (Debelle y Fisher, 1994; Posen, 1995; Walsh, 1994); la menor inflación, por tal razón, se logra a costa de un algún grupo. En las economías en desarrollo, las diferencias en la IBC estatutaria 
no parecen predecir la inflación, porque algunos gobiemos aplican una política monetaria inflacionaria independientemente de los estatutos (Cukieman, 1992; Cukiermán, Webb y Neypati, 1992), lo que implica que todavía creen en las ganancias de una mano libre ${ }^{3}$. En otras palabras, la IBC no satisface a todas las personas todo el tiempo, porque esta opción de régimen monetario tiene consecuencias distributivas.

Si existen consecuencias distributivas, no hay razón alguna para suponer que la adopción de la IBC se aplique por sí sola. Las preferencias por la estabilidad de precios que supone la IBC requieren respaldo político. Si la IBC no incluye dichas preferencias, no afectará la inflación a largo plazo; si dichas preferencias cuentan con un respaldo universal, la independencia sería innecesaria. El cambio de responsabilidad de jure para poner en práctica estas preferencias, no elimina la posibilidad de que no se aplique. Como dice McCallum (1995), “ciertamente, si la ausencia de una tecnología previa al compromiso es el problema, entonces debe aplicarse al banco central consolidado como entidad gubernamental, así como si se tratara de un banco central totalmente independiente". Ésta es la razón por la cual es tan importante ver qué fuerzas sociales apoyan la estabilidad de precios y pueden asegurar contra el riesgo que afecta a las instituciones monetarias, así como también examinar las instituciones monetarias mismas.

En este artículo se sostiene que el sector financiero está en una posición única para proporcionar este apoyo, y los bancos centrales han sido más independientes cuando ese respaldo ha sido mayor. La IBC recibe el apoyo del sector financiero como una forma, a largo plazo, de lograr la estabilidad de los precios, para la cual ese grupo de interés no tiene claro sustituto, pero no podría ser efectivo sin la protección constante del grupo para sus actividades antiinflacionarias. En consecuencia, el otorgamiento de la IBC en aquellos casos en que ni el sector financiero o alguna otra fuerza política estén dispuestos a defenderlo en momentos de costosa desinflación, es poco probable que tenga el efecto antiinflacionario deseado a largo plazo. Además, los cambios en el sistema financiero o político de un país probablemente alteran la capacidad del banco central para procurar alcanzar la estabilidad de los precios.

El argumento se presenta en cuatro partes. En la sección 2 se analizan las razones para que las políticas de los grupos de interés y el sector financiero, en particular, desempeñen una función en la política monetaria. En la sección 3 se analizan los elementos de la oposición financiera efectiva a la inflación, y se crea una medida compuesta de los mismos. En la sección 4 se verifica la predicción de que la IBC - y la baja inflación- es el resultado de una oposición financiera efectiva; primero en una muestra de 17 países de la OCDE, desde 1950 a 1989, utilizados en investigaciones anteriores sobre la IBC; luego en una muestra más amplia que incluye a estos países y 15 países en desarrollo con una 
inflación baja y moderada desde 1960 a 1989. La sección 5 analiza las implicaciones específicas para la evolución actual del argumento que se expone en este trabajo.

\section{Un modelo de intereses de política monetaria}

¿Por qué son importantes los intereses y las instituciones? Obviamente, en un determinado país algunas instituciones gubemamentales estarán más sujetas que otras al riesgo de cambio, estando más afirmadas las organizaciones consagradas en la constitución. Sin embargo, incluso la Corte Suprema de Estados Unidos ocasionalmente ha ajustado sus decisiones por razones de seguridad ${ }^{4}$. El examen de las instituciones también debe considerar el marco cronológico. En un análisis de períodos de tiempos más cortos, las instituciones y sus efectos probablemente se mantienen fijos. Como se buscan explicaciones para los patrones que persisten a lo largo de períodos más prolongados - tales como las diferencias promedio por década en las tasas de inflación que se investigan en este ensayo ( $y$, en general, en los debates relacionados a la IBC)—, deben tomarse en cuenta las probables fuentes de cambio institucional ${ }^{5}$.

Así, a pesar de que las decisiones sobre política monetaria de un banco central reflejan su capacidad institucional y sus limitaciones estatutarias, estas decisiones también responden a la realidad política que determina su autonomía y sus poderes $^{h}$. Este punto de vista general sobre la formulación de políticas monetarias se basa en cuatro supuestos de base empírica: en primer lugar, la inflación tiene significativos efectos redistributivos, con grupos identificables que resultan más perjudicados por la inflación; en segundo lugar, los grupos más perjudicados (por ejemplo, los que más se benefician de la estabilidad de precios) desempeñan el papel más prominente como grupos de presión en materia de política monetaria; tercero, ciertas estructuras políticas nacionales determinan la influencia de la presión de los grupos de interés sobre la política monetaria; y, por último, sin una efectiva presión de quienes se oponen a la inflación, el costo político de la desinflación podría hacer que los gobiernos presionen a los bancos centrales para que apliquen políticas menos estrictas. La independencia del banco central deriva de los deseos de un grupo de interés, que está más comprometido en favor de la estabilidad de precios que lo que el votante medio está decidido a reducir el riesgo del banco central en la búsqueda de políticas antiinflancionarias.

La independencia del banco central puede asegurar la estabilidad de precios cuando la oposición efectiva a la inflación la establece la meta de la politica ${ }^{7}$. A cualquier grupo interesado le resulta política y prácticamente imposible negociar directamente con el gobierno cambios en el nivel de precios: un banco central ofrece la experiencia, la capacidad operativa y la legitimidad política necesarias para conducir la política monetaria. En resumen, ni la voluntad políticamente efectiva de oponerse a la inflación ni los medios estatutarios para perseguir esta meta, pueden, por sí solos, lograr la estabilidad de precios. La oposición finan- 
ciera efectiva a la inflación y la IBC, juntos, son suficientes. En términos prácticos, esto significa que la IBC debería aumentar y la inflación debería disminuir, a medidś que aumenta la eficacia antiinflacionaria de un grupo de interés en proteger una política monetaria antiinflacionaria.

Por supuesto, parece limitante aislar cualquier grupo de interés como la fuente principal de una oposición efectiva a la inflación en todos los países. En cualquier sociedad, una coalición diferente de intereses podría (buscar) presionar en favor de la estabilidad de precios, incluyendo a los pequeños ahorristas y pensionistas, importadores y acreedores hipotecarios. La opción se ve facilitada por la existencia, en esta arena política, de un grupo de interés históricamente prominente: el sector financiero.

Las ventajas de organización de este sector sobre todos los otros grupos de interés en la discusión política de las instituciones de política monetaria son significativas. El sector financiero logra acceso al gobiemo elegido y a los funcionarios del banco central por ser sus principales fuentes extermas de información y asesoramiento en materia de política monetaria. Incluso, en aquellos países en donde los bancos no son propietarios y nominan directores aparte del banco central (como sucede en Estados Unidos), o mantienen reuniones regulares entre los representantes de las asociaciones del sector financiero y los responsables de las decisiones del banco central (como ocurre en Alemania), la vigilancia que el sector financiero ejerza sobre las acciones y el personal ejecutivo del banco central constituye una función prominente en la evaluación gubernamental de la política del banco central. Esto se logra a través de declaraciones públicas, interacción personal, empleo de antiguos funcionarios de bancos centrales y la provisión de parte de la oferta de nominados para gobernadores de los bancos centrales.

Además, desde hace mucho los intereses financieros han revelado una preferencia por la estabilidad de los precios. Como se sostiene en un informe sobre el debate del Congreso anterior relacionado con los cambios propuestos en el Sistema de la Reserva Federal de Estados Unidos:

Si la meta es minimizar la inflación, los funcionarios federales razonan, una manera segura de lograr esta meta será que los banqueros privados -que figuran entre los más belicosos en materia de inflación - designen a los presidentes de los bancos regionales ${ }^{\mathbb{b}}$.

Los economistas académicos han hecho supuestos similares. Lohmann (1992) interpreta a Rogoff (1985) de la siguiente manera:

Empíricamente, el nombramiento de un banquero central con una estrictamente positiva (es decir, un mayor peso relativo sobre la estabilización de la inflación) podría corresponder a la selección de un banquero central del sector bancario conservador o deliberadamente permitir que el banquero central sea captado por la comunidad financiera". 
La vunerabilidad de los banqueros frente a la inflación, aunque no completamente explicada, está bien establecida. Santoni (1986) encuentra que para el centro monetario de Estados Unidos y los bancos más pequeños, tanto la inflación no anticipada como la variación de la inflación anticipada tienen significativos efectos negativos en los precios de las acciones de los bancos (más que en el caso de la mayoría de las acciones), desde 1962:1 hasta 1985:4'1". Además, los bancos y el sistema financiero más amplio tienen mucho que temer de la desinflación. Los cambios bruscos en las tasas de interés, como los producidos por una política monetaria más restrictiva, pueden disminuir significativamente las utilidades (según lo ilustran los problemas recientes en el sector financiero estadounidense desde que la Reserva Federal comenzó a restringir la política en febrero de 1994).

\section{Una medida de oposición financiera efectiva a la inflación}

Para establecer la relación presentada aquí entre la oposición financiera efectiva a la inflación (de aquí en adelante OFI) y la IDC, y entre la OFI (a través de su efecto sobre la IBC) y la inflación, deberá diseñarse una medida de OFI consistente a nivel nacional. Tal medida requiere una mejor comprensión de las diferencias nacionales en los sistemas financieros y políticos. En particular, se tienen que responder dos preguntas: ¿Qué es lo que determina la unidad de la aversión del sector financiero por la inflación? ¿Qué es lo que hace que el sector financiero sea más efectivo al oponerse a la inflación?

Siguiendo a Olson (1965), es probable que los actores interesados se involucren más en la acción política colectiva cuando los beneficios para cada actor excedan el costo que les representa contribuir al esfuerzo, y cuando la participación de los miembros pueda vigilarse. Por tal razón, es importante establecer qué es lo que determina la aversión del sector financiero de un país por la inflación y su capacidad de acción colectiva, ya que no todos los sectores financieros de todos los países son igualmente vulnerables a verse perjudicados por la inflación. A medida que un mayor número de empresas financieras comparten un punto de vista similar, se reduce el costo de perseguir la meta del grupo y vigilar la participación, y el sector financiero se convierte en grupo de presión que se vuelve más viable.

Este énfasis en la unidad lleva a la primera fuente de variación entre países en el vigor del interés financiero en la estabilidad de los precios: los sectores financieros con las actividades bancarias globales deberán expresar sentimientos antiinflacionarios más fuertes que aquéllos que no las tienen. En la medida en que los clientes cambian fuentes de capital externo cuando cambia la inflación (digamos de deuda a capital, como en Fisher, 1982), los sistemas bancarios universales permiten que este cambio ocurra dentro de las empresas financieras, así que no existe una división competitiva entre los subsectores financieros en su 
reacción frente a la inflación. Es más, los sistemas bancarios universales tienen un camino organizacional más fácil al consenso político sobre todos los temas, ya que enfrentan reglamentaciones y mercados unificados.

El número de metas que el grupo de interés persigue es otra fuente de efectividad diferencial en la oposición del sector financiero a la inflación, especialización que reduce los costos: los sectores financieros que están menos bajo el poder regulatorio de un banco central deberian expresar una mayor oposición a la inflación que aquéllos que están bajo la mira del banco central. Cuando la autoridad monetaria restringe la asignación del crédito o de tasas de interés sobre préstamos, o cuando tiene una responsabilidad de supervisión de los bancos, las firmas individuales podrían entrar en conflicto con el banco central. Podrían buscar limitar los poderes del banco central; podrían presionar por un relajamiento general del crédito para eludir determinados límites reglamentarios, a pesar del efecto inflacionario; o podrían librar batallas individuales con el banco central. Por el contrario, cuando el banco central no tiene responsabilidad de supervisión bancaria, el sector financiero no se desvía de este apoyo a la autonomía del banco central.

Podría parecer un poco contrario a la intuición decir que es mejor para la búsqueda de la independencia del banco central el hecho de que tiene una influencia menos directa sobre los posibles aliados. Cuando el sector financiero interactúa con el banco central sólo en lo relacionado con los arreglos monetarios , sin embargo, la única pérdida potencial son las actividades de cabildeo que no recuperan los costos. Al permitir que el banco central ejerza presión sobre el sector financiero más allá de la comunidad de intereses sobre la estabilidad de precios, se cambian las oportunidades del sector. En ese caso sería preferible que el sector financiero dedicara sus recursos de cabildeo en materia de políticas a reducir la influencia del banco central, en vez de buscar una suficiente IBC.

Cuando se mide la apertura del sistema político a la influencia de los grupos de intereses, es preciso saber quién decide sobre las metas de política dentro del sistema y quien las implementa. Las respuestas pueden considerarse en términos de la disciplina legislativa de un sistema político y su centralización en materia de ejecución de políticas. La disciplina caracteriza la medida en que los altos funcionarios elegidos del gobierno (primeros ministros, líderes de partido) pueden lograr que la legislatura apruebe sus iniciativas sin alteraciones. La centralización es la clave de cómo se pone en práctica la política diaria, ya sea directamente por los funcionarios elegidos o por burócratas que han recibido esta delegación o aun indireclamente por grupos privados.

El concepto de disciplina legislativa deriva de la visión de Buchanan y Tullocks (1962) del proceso legislativo en las democracias. Los legisladores con intereses propios extraerán de los iniciadores de cualquier propuesta legislativa 
pagos laterales hasta el valor de los votos de los iniciadores, incluso si la propuesta sirve a sus propios intereses. Cuando las partes son menores en número, o la disciplina partidaria es mayor, el número de puntos de veto (y posibles fuentes de enmiendas) es menor; cuando las partes aumentan en número, se incrementa la importancia de los miembros individuales, así como el acceso a las decisiones legislativas ( $y$, por consiguiente, las componendas).

La medida básica generalmente invocada por los científicos políticos para caracterizar la diversidad y el número de partidos en una legislación es la fraccionalización, definida como la probabilidad de que dos legisladores seleccionados al azar pertenecerán a diferentes partidos ${ }^{12}$. A medida que aumenta la fraccionalización, los intereses locales vinculados a determinados partidos o a los electorados de ciertos miembros deberían ver sus metas particulares mejor servidas por el proceso legislativo. A medida que disminuye la fraccionalización y las decisiones legislativas se mueven a los líderes de los partidos nacionales (cuyo número es menor), los intereses organizados a nivel nacional que ocupan el tiempo de estos líderes tendrán más voz ${ }^{13}$. Dado el interés del sector financiero en un asunto nacional (la estabilidad de precios) y alegatos de experiencia (cuando hablamos de "confianza" de mercados financieros en las diversas medidas), podemos predecir que donde el sistema partidario de un país está menos fraccionalizado, la oposición financiera a la inflación debería ser más efectiva.

De manera similar, la centralización de la ejecución de las políticas se mide por el acceso que tengan a los legisladores otros intereses diferentes de los del gobierno elegido después que haya aprobado la legislación inicial. Cuanto más delegue el gobierno la ejecución de las políticas, más permitirá que los resultados de política sean divergentes de sus intenciones, en gran parte debido a las dificultades de vigilancia. Además, ya que la implementación de las políticas siempre involucra una gran discrecionalidad en la interpretación y la reacción ante los acontecimientos actuales, una política descentralizada podría forzar a las instituciones ejecutoras a que recurran a recursos diferentes de los que podría haber escogido el gobierno elegido para información y apoyo. En un sistema descentralizado, los grupos de intereses privados cuentan con más formas de influir sobre la política.

La forma más marcada de descentralización de un sistema político es estructurarlo como un estado federal. Si bien esta decisión generalmente es motivada por razones étnicas o regionales, tiene el efecto general de aislar lo que se asigna como tema puramente nacional - tal como la política monetaria - de muchas otras políticas y de estimular formas de delegación para la implementación de políticas. Además, el federalismo niega el acceso a los temas nacionales, excepto a aquellos grupos - como el sector financiero- que pueden ofrecer un frente unificado a través de las regiones. Aunque el federalismo tal vez no sea una condición necesaria para la descentralización de políticas, es una condición suficiente. 
Por tal razón, la oposición financiera a la inflación debería ser más efectiva en los sistemas federales de gobierno. El diseño y la creación de los Bank Deutsches Länder (bancos alemanes regionales), la base del Bundesbank, en la Alemania Occidental de la postguerra, ilustra este punto. Las fuerzas de ocupación de Estados Unidos estimularon al gobierno alemán a evitar darle poder al gobierno central alemán y afirmar el poder de la sociedad civil (es decir, los intereses privados y las asociaciones) en todos los arreglos sobre nuevas instituciones. Cuando la reforma monetaria de 1948 requirió la creación de un nuevo banco central para reemplazar al difunto Reichsbank, fue deliberadamente organizado según un sistema federal, con canales institucionales para escuchar al sector financiero. Este mismo enfoque fue adoptado en la creación del Sistema de la Reserva Federal de Estados Unidos por quienes estaban preocupados por el poder centralizado.

En resumen, la efectividad de la oposición del sector financiero a la inflación debería verse como resultado de la unidad de su interés en la estabilidad de los precios, y la apertura del sistema político del país a la influencia de los grupos de intereses. Al igual que con la IBC, la medición de los factores económicos y políticos cualitativos que contribuyen a la OFI se hace mejor sin ilusiones de precisión. Para las investigaciones estadísticas de la siguiente sección, el autor ha preparado un índice de oposición financiera efectiva a la inflación - denominada OFI efectiva - compuesto de las medidas siguientes:

Univbk $=1$, si los bancos pueden comercializar por lo menos dos tipos de valores, seguros y préstamos comerciales, $=0$ de otra manera ${ }^{14}$.

Regpow $=1$, si la supervisión bancaria no está bajo el banco central; = $0.5 \mathrm{si}$ es compartida por el banco central y otro organismo; $=0$ si es solamente la responsabilidad del banco central ${ }^{15}$.

Federal $=1$, si un país tiene una estructura política federal; $=0$ en caso contrario $^{16}$.

Fractn = la probabilidad de que dos legisladores, escogidos al azar, de una legislatura nacional pertenezcan a diferentes partidos ${ }^{17}$.

De acuerdo con el análisis de esta sección, un mayor valor de cada uno de los tres componentes se supone que representa una unidad mayor (univbk, regpow) o efectividad (federal) de oposición financiera a la inflación; mientras que un mayor valor de Fractn se supone que disminuye la efectividad. Este índice de la OFI efectiva se fija igual a Univbk + Regpow + Federal - Fractn, y puede oscilar entre -1 y 3 . Cuanto mayor sea el valor de la OFI efectiva, indica una oposición financiera más efectiva a la inflación en ese país.

Utilizando estas definiciones, se determinaron valores para el índice de la OFI efectiva y sus componentes para un grupo de 32 países con inflación baja a moderada, por décadas, desde 1960 a $1989^{18}$. Se incluyeron diecisiete países de 
la OCDE (Australia, Austria, Bélgica, Canadá, Dinamarca, Francia, Alemania, Grecia, Irlanda, Italia, Japón, Países Bajos, Nueva Zelandia, España, Suiza, Reino Unido y Estados Unidos) ${ }^{19}$, así como 15 países en desarrollo de Asia y América Latina que no sufrieron hiperinflación durante más de una década del período examinado (Bolivia, Chile, Colombia, Hong Kong, India, Indonesia, Malasia, México, Pakistán, Filipinas, Singapur, Corea del Sur, Tailandia, Turquía y Venezuela) ${ }^{211}$.

Las observaciones en las que la tasa de inflación promedio de una década en un país exceden el 30 por ciento anual (Indonesia en los años sesenta, Chile en los setenta y Bolivia, México y Turquía en los ochenta) se excluyeron de la muestra. El índice de la OFI efectiva para las otras noventa observaciones tiene un valor medio de 0,92 , una desviación estándar de 0,78 , y un valor mínimo de $-0,48$ (Corea del Sur en los años setenta), y un valor máximo de 2,81 (Bélgica en los años ochenta). Las observaciones de países durante períodos en los que los niveles de inflación promedio se caracterizaron por explosiones hiperinflacionarias están excluidos, y se cree que son situaciones en que el proceso y el apoyo a la desinflación probablemente difieran del curso descrito en este trabajo ${ }^{21}$.

Volviendo a los diecisiete países de la OCDE codificados (una submuestra útil para la comparación con la literatura anterior sobre la IBC) y extendiendo hacia atrás el índice de los años cincuenta, hay 68 observaciones de OFI efectiva, con un valor medio de 1,05 , una desviación estándar de 0,86 y un rango de un valor mínimo de $-0,23$ (Italia durante toda la época) a un máximo de 2,81 (de nuevo Bélgica en los años ochenta). La distribución de la OFI efectiva en la submuestra de países de la OCDE y en la muestra mayor no parece diferir sustancialmente.

\section{El índice de la OFI efectiva como variable predictiva de la independen- cia del banco central y la inflación}

Existen evidencias estadísticas claras en favor de un vínculo causal (más que una mera asociación) entre una OFI más efectiva, por un lado, y una IBC mayor y tasas de inflación menores, por el otro. El índice de la OFI efectiva resulta ser una significativa variable predictiva de la IBC. Aunque esto solamente es una correlación, es difícil imaginarse un argumento causal de primer orden que pueda obtenerse de la IBC $a$ los varios componentes de la OFI efectiva, de manera que supuestamente se mantiene el argumento contrario. Además, la OFI predice tasas promedio de inflación aunque la IBC no lo hace, cuando ambos factores son tomados en cuenta. La IBC comúnmente se supone que tiene la habilidad de disminuir la inflación, independientemente del contexto de política del banco central, y no está respaldada por los datos.

Una medida de la IBC se utiliza durante toda la sección: la medida "LVAU" (variables legales agregadas no ponderadas de Cukind, Cukierman, 1992). Los 
otros índices publicados de IBC estatutaria -Bade y Parkin, 1988; Grilli, Mansciandaro y Tebellini, 1991; Alesina y Summers, 1993, promedian estos dos en una eścala de cuatro puntos - no parecen ser tan confiables como el índice de Cukierman, que va a un nivel mucho más fino de detalle y capta la variación limitada aunque real de la IBC a lo largo del tiempo. De hecho, el índice de Cukierman muestra una mayor correlación con la inflación en comparación con las muestras de los otros autores que sus propios índices de IBC (Cukierman, Webb y Neyapti, 1992). Además, Cukind se ha computado y calculado para una amplia gama de país, permitiendo el análisis de las relaciones entre OFI, IBC y la inflación más allá del subconjunto de países de la OCDE normalmente examinado ${ }^{22}$.

Los efectos previstos de la OFI efectiva -que la IBC es mayor cuando la OFI es mayor, y que la inflación es menor cuando la OFI es mayor-se prueban examinando un panel transnacional formado por observaciones de décadas de las OFI y sus componentes, Cukind e inflación (calculada de cifras del IPC en Estadisticas Financieras Internacionales del FMI) para los 32 países de la muestra de este trabajo. En la muestra completa, Cukind tiene una media de 0,36 , con una desviación estándar de 0,16 (Cukind está diseñado para funcionar de 0 a 1), mientras que la inflación tiene una media de 7,8 por ciento por año, con una desviación estándar de 5,5 por ciento. En la submuestra de la OCDE, Cukind tiene una media de 0,39, con una desviación estándar de 0,16 , mientras que la inflación media es de 5,3 por ciento, con una desviación estándar de 3,1 por ciento.

La hipótesis nula es la supuesta por la literatura hasta la fecha sobre la IBC: que la IBC no es significativamente endógena a la oposición financiera efectiva a la inflación (o a cualquier otro factor). Como evidencia a favor del modelo de política monetaria de este ensayo, tomaré un coeficiente significativamente positivo de OFI en ecuaciones estimando la IBC, y coeficiente significativamente negativos de OFI en ecuaciones calculando la inflación cuando la IBC también se incluye como variable explicativa. Además, todas las ecuaciones están estimadas con los componentes de OFI como variables explicativas; los coeficientes de Univbk, Regpow y Federal se predicen con el mismo signo que la OFl efectiva, mientras que el coeficiente Fractn podría tener el signo opuesto.

Para neutralizar el efecto de los factores más allá de las interacciones de nuestras variables de interés, se introducen variables indicativas para tener en cuenta los shocks específicos por décadas entre países y para efectos regionales. Además, se incluye una variable igual a 1 para cualquier década en la cual el país era miembro de un régimen de tipo de cambio fijo durante más de cinco años, y cero en caso contrario, en regresiones en las que la inflación es la variable dependiente. Como la inflación muestra una tendencia ascendiente a lo largo del período 1950-1989, aun después de neutralizar el efecto de los shocks 
específicos por década, se estimaron todas las ecuaciones en que la inflación es una variable dependiente mediante mínimos cuadrados ponderados, donde las observaciones son ponderadas por un número índice por período (por ejemplo, años cincuenta $=1$, años sesenta $=2$ ). Todas las ecuaciones en las que la IBC es una variable dependiente se calculan mediante mínimos cuadrados ordinarios.

La prueba principal del argumento de este trabajo es determinar la capacidad de la OFI para explicar niveles de IBC y tasas de inflación en la misma muestra de países de la OCDE, que aquella usada para establecer la correlación negativa entre la IBC y la inflación (Grilli, Masciandaro y Tabellini, 1991; Alesina y Summers, 1993). De acuerdo con la literatura sobre la IBC, la Gráfica 1 traza la IBC y la inflación contra la OFI para los valores promedios de los diecisiete países durante el período 1950-1989. Las relaciones previstas aparecen claramente.

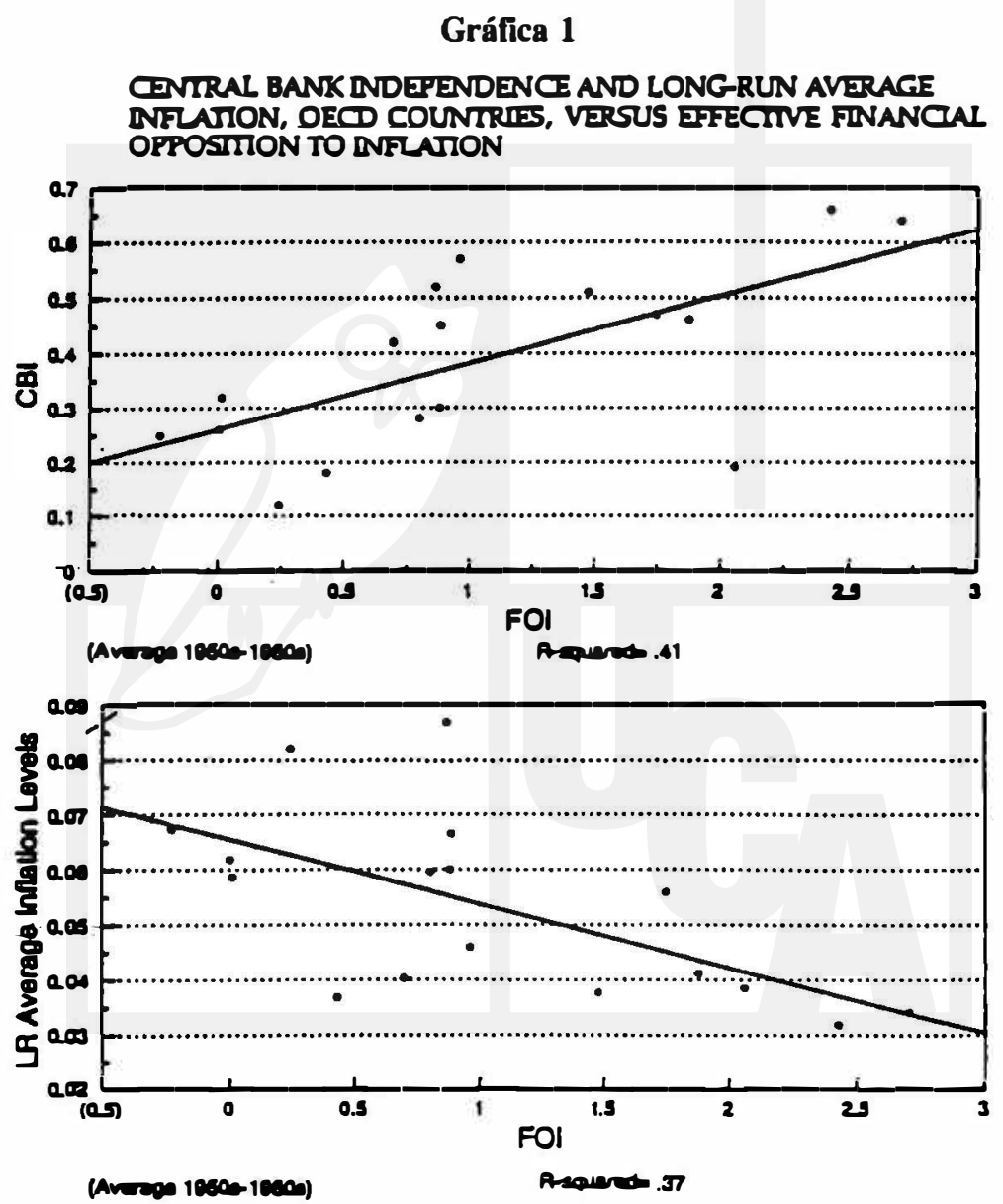


Volviendo a exploraciones más formales de las relaciones en cuestión, el Cuadro 1 muestra los resultados de las regresiones OLS de Cukind sobre OFI efectiva $y /$ sus componentes, con y sin variables por década. También se incluye la participación en un régimen de tipo de cambio fijo en caso de que esta forma de compromiso del gobiemo en favor de la restricción monetaria se sustituya por la IBC $^{23}$. Los resultados respaldan fuertemente el papel de los intereses financieros en la determinación de la IBC. El coeficiente de OFI efectiva es positivo y significativo al nivel del 1 por ciento en ambas ecuaciones (I y III) cuando aparece. En las ecuaciones II y IV, cada coeficiente del componente OFI tiene el signo previsto y todas, excepto Regpow, son significativas al nivel del 1 por ciento en ambas ecuaciones. Para tener algún sentido de las magnitudes, nótese que un aumento de 1 en la desviación estándar en la OFI aumentará el índice de Cukind en cerca de $2 / 3$ de la desviación estándar $(0,10)$.

\section{Cuadro 1}

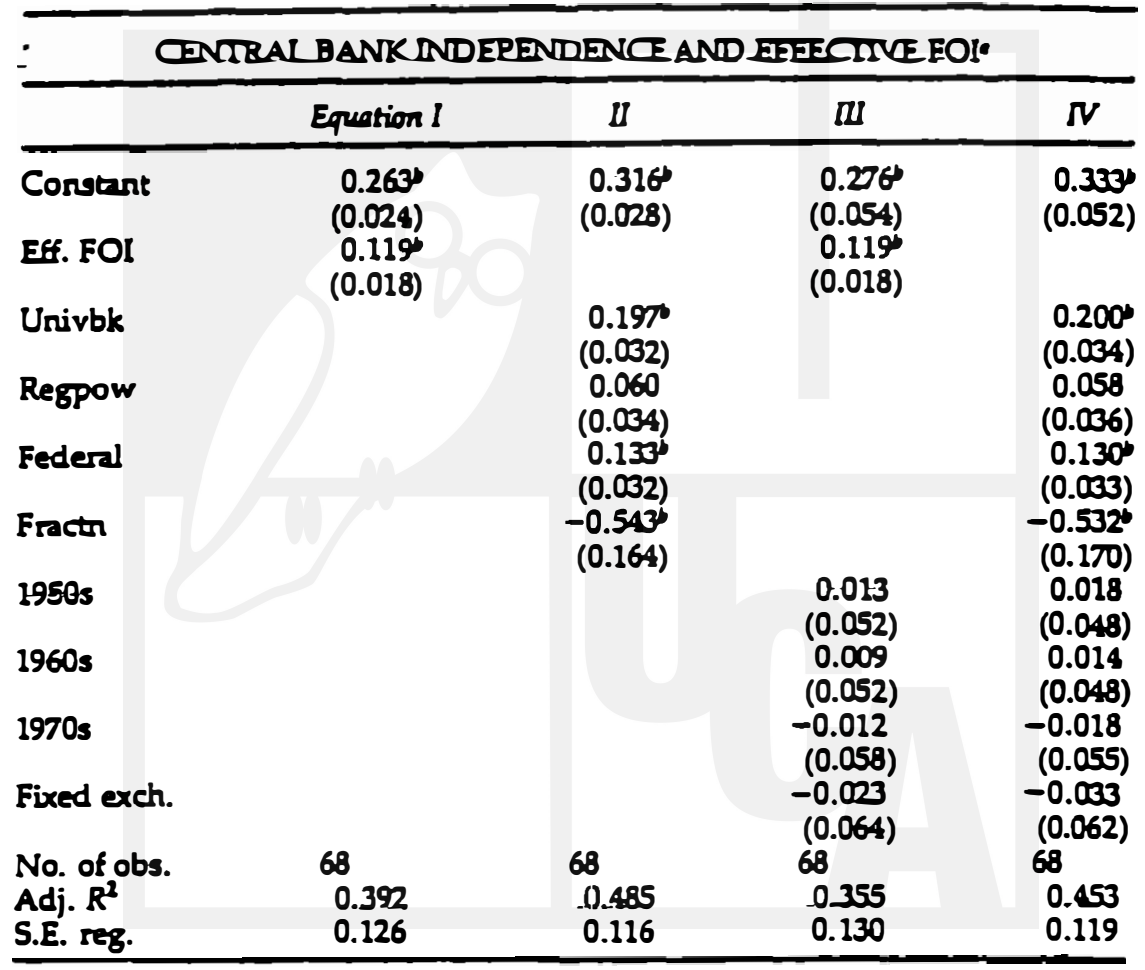

- Otacervations - averages by deade, 1950-1999, 17 OECD countries. Dependent variabier centud benk independenco LVAU (Cukieman. 19\%2). Estimated by ordinay lenst squar. Sandard erors gven in parenthese.

Sigrifont at the 1\% level. 
Dada la imagen clara del Cuadro 1, no deben sorpender las evidencias del Cuadro 2 para las predicciones de este trabajo. El coeficiente la OFI efectiva es negativo y significativo al nivel del 1 por ciento en las tres ecuaciones que estiman la inflación por mínimos cuadrados ponderados (en función del tiempo) donde aparecen (V, VII, IX) ya sea que se incluyan o no Cukind, la participación en regímenes de cambio fijo, o los efectos por décadas. En las otras tres ecuaciones estimadas, los coeficientes de todos los componentes de la OFI tienen el signo previsto, siendo los coeficientes Regpow y federal significativos al nivel del 5 por ciento o más en cada ecuación.

Cuadro 2

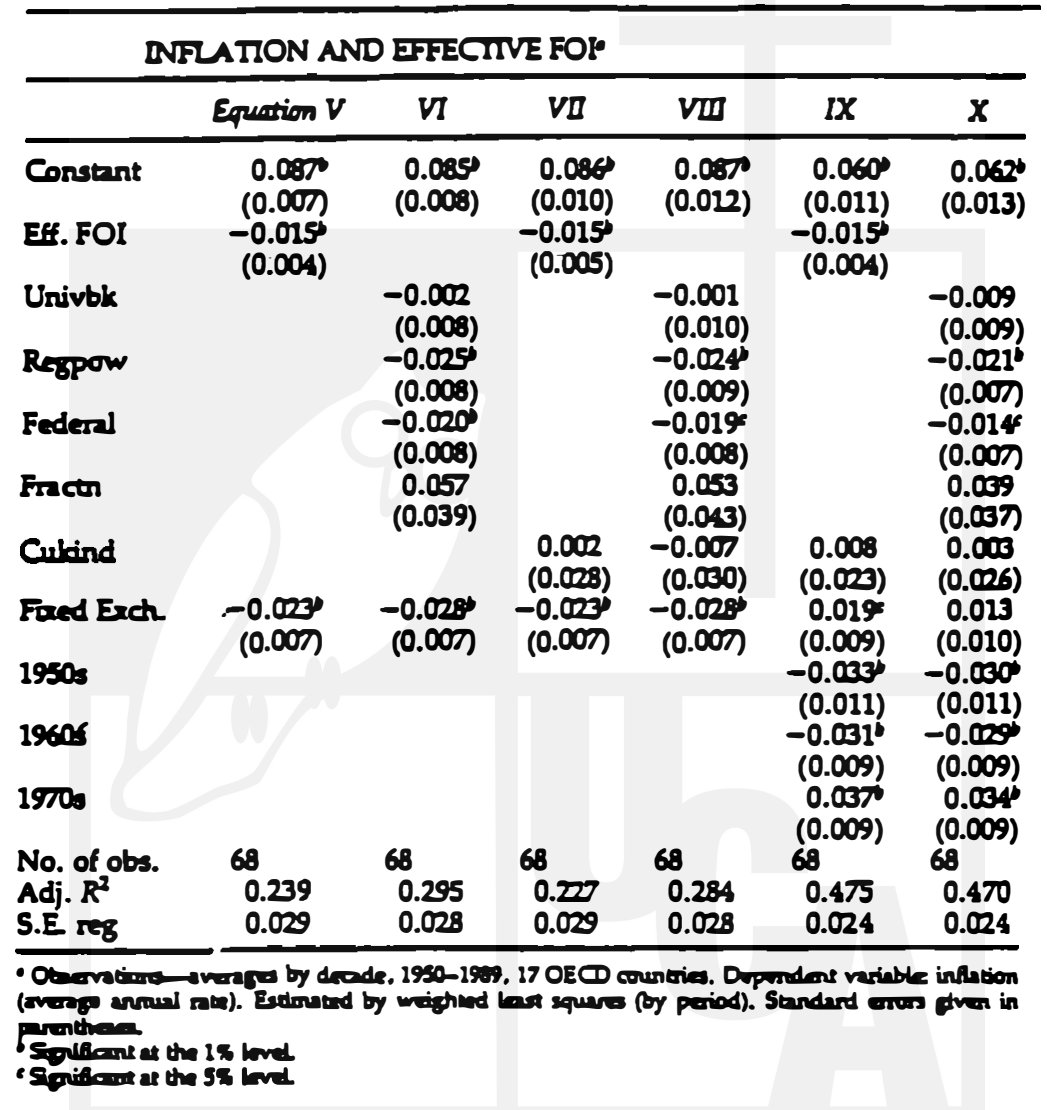

Cukind nunca tuvo un coeficiente significativo en las ecuaciones que predicen la inflación cuando se incluye la OFI efectiva y muestra un coeficiente positivo en tres de las cuatro ecuaciones, contrariamente a las expectativas de la literatura sobre instituciones. Los valores ajustados de $\mathrm{R}^{2}$ para las ecuaciones $\mathrm{V}$ $\mathrm{X}$, son comparables a los de las regresiones publicadas de inflación sobre IBC 
sin OFI. El panorama ciertamente parece ser uno en que el índice OFI capta la correlación con la inflación normalmente asociada con la IBC. Un aumento de una desviación estándar en la OFI efectiva reduce la inflación en 1,3 por ciento anual, cerca del 40 por ciento de una desviación estándar.

Cuadro 3

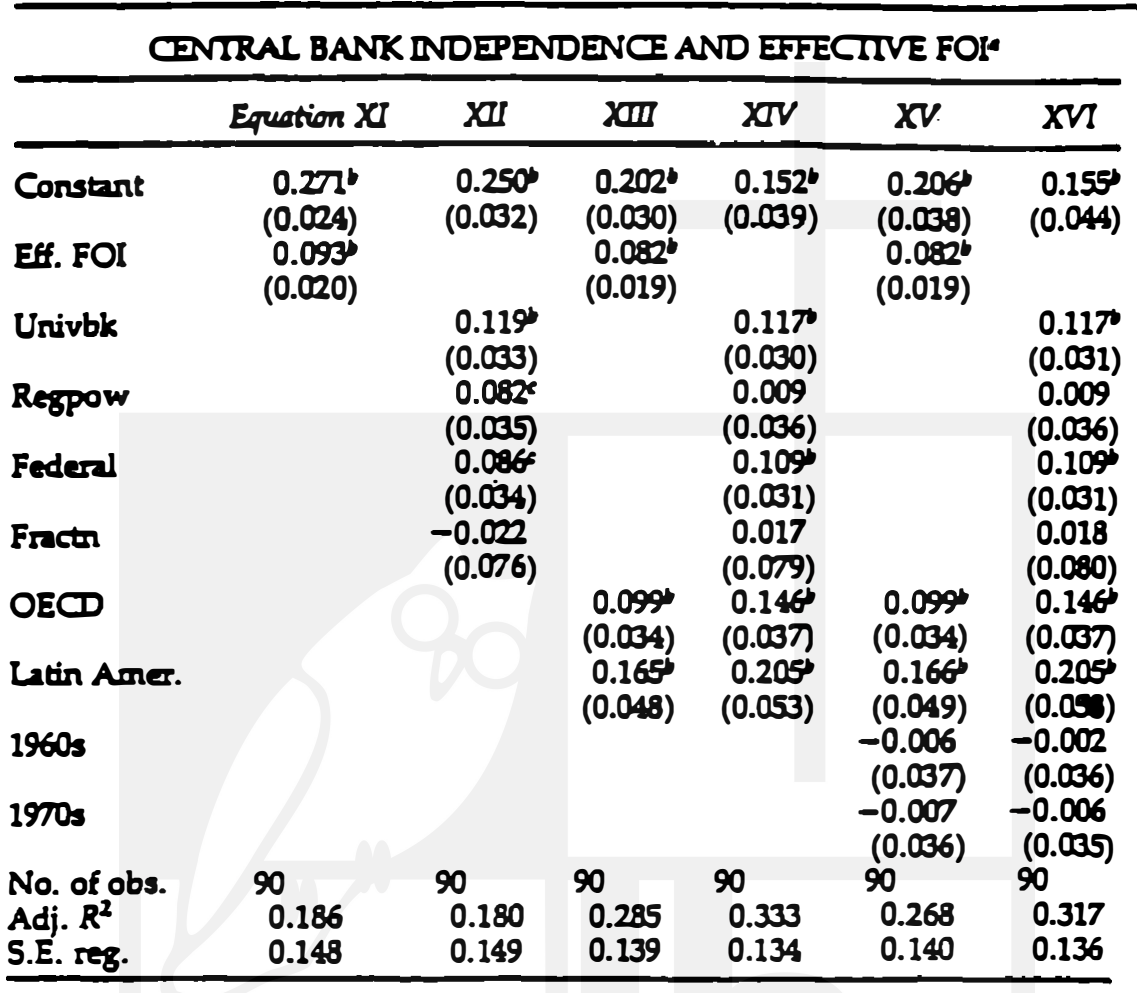

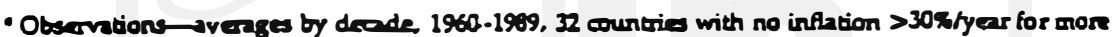

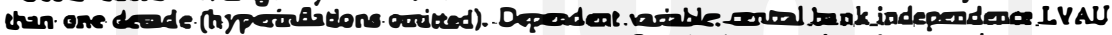

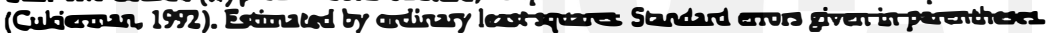

- Siguifiant at the 1\% lovel

- Sigifiant at the 5\% lovel

¿La relación entre OFI, IBC e inflación es específica de las democracias industrializadas? Este aspecto es de interés no solamente para asegurarnos de que la relación entre OFI, IBC e inflación no es tanto un artefacto de la particular muestra de la OCDE examinada, sino para averiguar la aplicabilidad de la IBC como norma para los arreglos monetarios en economías en transición y en desarrollo (como muchos abogan). Hasta ahora, el único examen estadístico de la IBC y la inflación, que incluye países en desarrollo en la muestra ${ }^{24}$, indica que la IBC estatutaria no es efectiva contra la inflación fuera de la OCDE. 
Cuadro 4

DNRLATION AND EFFECTVE FOI-

\begin{tabular}{|c|c|c|c|c|c|c|}
\hline & $\begin{array}{l}\text { Equation } \\
X V I I\end{array}$ & $x$ & $x x$ & $x x$ & XOI & $x \mathbf{X I I}$ \\
\hline $\begin{array}{l}\text { Constant } \\
\text { Eff. FOI }\end{array}$ & $\begin{array}{r}0.07 \% \\
(0.014) \\
-0.018 \% \\
(0.008)\end{array}$ & $\begin{array}{c}0.0635 \\
(0.015)\end{array}$ & $\begin{array}{r}0.086 \\
(0.013) \\
-0.013 \\
(0.007)\end{array}$ & $\begin{array}{c}0.085 b \\
(0.015)\end{array}$ & $\begin{array}{r}0.076 \\
(0.015) \\
-0.013 \\
(0.006)\end{array}$ & $\begin{array}{r}0.080 \% \\
(0.013)\end{array}$ \\
\hline Univbk & & $\begin{array}{c}-0.004 \\
(0.012) \\
-0.001\end{array}$ & & $\begin{array}{r}0.004 \\
(0.011) \\
-0.008\end{array}$ & & $\begin{array}{c}0.000 \\
(0.010) \\
-0.008\end{array}$ \\
\hline Federal & & $\begin{array}{r}(0.01 \\
-0.03 \\
(0.01\end{array}$ & & $\begin{array}{r}(0.012) \\
-0.0305 \\
(0.011)\end{array}$ & & $\begin{array}{r}(0.011) \\
-0.025 \\
(0.010)\end{array}$ \\
\hline Fractn & & $\begin{array}{l}0.081^{6} \\
(0.009)\end{array}$ & & $\begin{array}{l}0.025 \\
(0.025)\end{array}$ & & $\begin{array}{l}0.032 \\
(0.024)\end{array}$ \\
\hline Cubind & $\begin{array}{c}0.073 \\
(0.099)\end{array}$ & $\begin{array}{l}0.057 \\
(0.037)\end{array}$ & $\begin{array}{r}0.049 \\
(0.037)\end{array}$ & $\begin{array}{l}0.041 \\
(0.037)\end{array}$ & $\begin{array}{c}0.043 \\
(0.034)\end{array}$ & $\begin{array}{c}0.050 \\
(0.033)\end{array}$ \\
\hline $\begin{array}{l}\text { Fuxed Exch. } \\
\text { OECD }\end{array}$ & $\begin{array}{c}-0.015 \\
(0.012)\end{array}$ & $\begin{array}{c}-0.016 \\
(0.011)\end{array}$ & $\begin{array}{c}-0.010 \\
(0.010) \\
-0.0248 \\
(0.012)\end{array}$ & $\begin{array}{c}-0.014 \\
(0.010) \\
-0.023 \\
(0.014)\end{array}$ & $\begin{array}{c}0.006 \\
(0.010) \\
-0.025 \\
(0.012)\end{array}$ & $\begin{array}{r}0.009 \\
(0.010) \\
-0.007 \\
(0.011)\end{array}$ \\
\hline Latiri Áner. & & & $\begin{array}{l}0.0648 \\
(0.018)\end{array}$ & $\begin{array}{l}0.0570 \\
(0.020)\end{array}$ & $\begin{array}{l}0.055^{\circ} \\
(0.018)\end{array}$ & $\begin{array}{l}0.065 \\
(0.016)\end{array}$ \\
\hline 1960 s & & & & & $\begin{array}{r}-0.038 \\
(0.012)\end{array}$ & $\begin{array}{r}-0.040^{\circ} \\
(0.012)\end{array}$ \\
\hline 1970s & & & & & $\begin{array}{c}0.0225 \\
(0.010)\end{array}$ & $\begin{array}{l}0.0265 \\
(0.010)\end{array}$ \\
\hline $\begin{array}{l}\text { IJo. of obs. } \\
\text { Adj. } R^{2} \\
\text { S.E. reg. }\end{array}$ & $\begin{array}{l}90 \\
0.045 \\
0.054\end{array}$ & $\begin{array}{l}90 \\
0.160 \\
0.051\end{array}$ & $\begin{array}{l}90 \\
0.303 \\
0.046\end{array}$ & $\begin{array}{l}90 \\
0.334 \\
0.045\end{array}$ & $\begin{array}{l}90 \\
0.464 \\
0.040\end{array}$ & $\begin{array}{l}90 \\
0.445 \\
0.041\end{array}$ \\
\hline
\end{tabular}

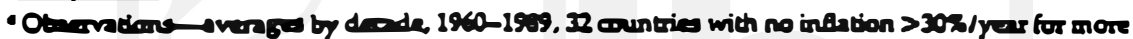

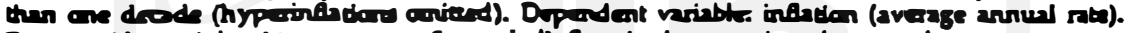

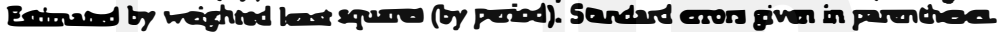

- Simifioud at at 15 lod.

- Signifous at the 55 lod

Las investigaciones estadísticas de esta sección muestran, sin embargo, que cuando el análisis se limita a aquellos países con tasas de inflación bajas o moderadas, independientemente de su situación de países en desarrollo, la relaciones previstas existen (aunque un poco más débiles que en la muestra de países de la OCDE). El Cuadro 3 muestra el resultado de repetir las estimaciones del Cuadro 1 para la muestra de 32 países de este trabajo entre 1960 y 1989, considerando los efectos comunes entre grupos de países (OCDE, Asia, América Latina).

La OFI efectiva tiene un coeficiente positivo y significativo (al nivel del 1 por ciento) en las tres regresiones (XI, XIII, XV) en las cuales aparece. Un 
aumento de 1 en la desviación estándar en la OFI efectiva (0,78 en esta muestra), lleva a un aumento de más del 40 por ciento de una desviación estándar en Cukind $(0,7)$. Univbk, Regpow y Federal tienen el signo positivo previsto en las tres ecuaciones en las cuales aparecen, y Univbk y Federal son siempre significativas al nivel del 5 por ciento o más. Como en el Cuadro 1, Regopow no parece ser independientemente importante para la IBC. A diferencia de lo que ocurre en la muestra de la OCDE, el efecto independiente de Fractn sobre la IBC desaparece (tal vez debido a que se incluyen países no democráticos en la muestra, lo que hace que sean menos importantes las estructuras partidarias).

El Cuadro 4 muestra los resultados de repetir las estimaciones del Cuadro 2 en una muestra más amplia, con variables predictivas adicionales que incluyen los efectos por grupos de países. La OFI efectiva siempre tiene el coeficiente negativo previsto, cayendo apenas por debajo del nivel del 5 por ciento de significación en la ecuación XIX, pero es significativa en las otras tres regresiones en las que aparece. Regpow, Federal y Fractn tiene el signo previsto en cada ecuación en la que aparecen, siendo el coeficiente de Federal significativo en todas las ecuaciones. De nuevo, Cukind nunca muestra un coeficiente negativo significativo (de hecho, el coeficiente siempre es positivo). La evidencia estadística continúa respaldando fuertemente las predicciones de este trabajo, aun cuando se incluyen en la muestra países que no pertenecen a la OCDE con inflación baja y moderada (pero no alta o hiperinflación).

Una distinción útil entre países en base a los niveles promedio de inflación es difícil de reconciliar con la literatura sobre la IBC. Cuando sólo se utilizan las instituciones monetarias como variables explicativas, no existe razón para pensar que la IBC ofrezca menos de un compromiso en favor de la estabilidad de precios para un país en puertas de una hiperinflación que para un país con una tasa de inflación del 10 al 20 por ciento, una vez que se realiza la transición entre los regímenes. La IBC debería predecir la inflación en todos los países (suponiendo alguna graduación razonable de los resultados de inflación) en ese marco. Cuando no es así, deben invocarse factores externos al modelo ${ }^{25}$ para justificar una distinción entre países que pertenecen o no a la OCDE, una distinción que los resultados de esta sección muestran como engañosos.

En contraste, la distinción empíricamente respaldada entre países por niveles de inflación resulta lógicamente del argumento ofrecido aquí. Un país con una inflación persistentemente alta o una hiperinflación, tendrán un sector financiero que habrá abandonado la oposición a la inflación hace mucho tiempo. Para sobrevivir, los bancos y otras empresas financieras tendrían que adaptarse al entorno monetario y tendrían que apoyar la inflación continuada. Y aquellos atributos de un sistema financiero y político de un país que aumentarían la oposición financiera efectiva a la inflación en un entorno menos inflacionario pasar a incrementar el apoyo a la inflación. Con este protector principal sumado a las 
fuerzas que favorecen una política menos restrictiva, un banco central independiente no puede sostener una política antiinflacionaria; una IBC estatutaria no tendrá ningún efecto sobre la inflación en este caso. El patrón de cómo se correlacionan negativamente los niveles de inflación con la IBC se explica a través de los incentivos que enfrenta el sector financiero.

Este patrón se ilustra mejor en el caso de Brasil, país que ha sufrido hiperinflaciones durante gran parte del período de posguerra. Los bancos brasileños han realizado grandes inversiones en automatización y sucursales locales para poder enfrentar los cambios de precios, comprometiéndose a una estructura adecuada para la hiperinflación. Han obtenido grandes utilidades de los préstamos al gobierno y han manejado agresivamente la flotación. De hecho, con la reciente introducción de la nueva moneda brasileña, el real, la rentabilidad bancaria disminuyó. Es importante señalar que Brasil es un estado federal, que tenía una banca universal en los años setenta y ochenta, y sólo una fraccionalización moderada de partidos. Brasil casi caería en una desviación estándar sobre la media del índice de la OFI. Tal vez no sea coincidencia que Brasil haya tenido menos éxito que sus vecinos en la lucha contra la hiperinflación ${ }^{2 n}$.

\section{Implicaciones: la política monetaria cuando los intereses delimitan las instituciones}

El argumento fundamental del autor - que los niveles de IBC reflejan las diferencias nacionales en la oposición financiera efectiva a la inflación- rechaza el supuesto de que el patrón de IBC observado antes de 1989 era aleatorio. Como resultado, los bancos centrales con independencia estatutaria comparable como cuestión de planificación, en vez de presión política ofrecerán grados significativamente diferentes de protección de la inflación a lo largo del tiempo a medida que se altera la situación política. Tanto la independencia del banco central como una coalición en la sociedad dedicada a proteger esta independencia, son necesarias para lograr la baja inflación adjudicada específicamente a la IBC; ninguna de las dos por sí sola es suficiente.

Se puede hacer una analogía entre el ingreso del Reino Unido al Sistema Monetario Europeo, en octubre de 1990, a la que demostró ser un tipo de cambio imposible de respaldar entre el marco alemán y la libra de 2,95, y su posterior salida en septiembre de 1992. El hecho de que el gobierno se hubiera atado las manos a través del SME no logró inducir cambios en la economía británica. El plan de crear una estabilidad de precios solamente hubiera funcionado en el caso inverso, en que los cambios estructurales efectuados hubieran permitido la adherencia al tipo de cambio.

El interés de análisis de este trabajo también implica que de aquí en adelante ciertos cambios aparentemente no relacionados en la organización de los mercados financieros o en el debate político afectarán las tasas de inflación. Desde el 
punto de vista financiero, la desreglamentación hacia la banca universal y la desintermediación por parte de las firmas financieras ejercen presiones conflictivas en los intereses del sector financiero de oponerse a la inflación. El resultado neto de estas tendencias tendrá efectos macroeconómicos influenciando el apoyo financiero a las políticas desinflacionarias. Desde el punto de vista político, la tendencia hacia la descentralización dentro de los países europeos y hacia el federalismo europeo, en general, aumentará la estabilidad de precios aislando las influencias financieras en los arreglos monetarios.

Es interesante notar, desde este punto de vista, que el efecto antiinflacionario del Banco Central Europeo propuesto sería bastante grande. Debido a que no será responsable de la supervisión financiera, el sector financiero rara vez entrará en conflicto directo con el mismo. Además, la mayoría abrumadora de los grupos de interés financiero nacionales en Europa ya lo apoyan explícitamente, en la esperanza de que suprimirá sus respectivas inflaciones nacionales. Como un símbolo de la unidad europea, y consagrado en un tratado internacional virtualmente sacrosanto, el Banco Central Europeo está protegido de los ataques a su estructura y existencia en la medida en que ningún banco central nacional lo ha estado ${ }^{27}$. Por último, el federalismo supranacional europeo debería limitar la influencia sobre las decisiones a los intereses más organizados y reconocidos.

Para el autor, las diferencias inflacionarias entre nacionales normalmente atribuidas a la IBC solamente no implican que la estructura de los bancos centrales sean irrelevante, o que el sector financiero sea la única influencia externa sobre la política monetaria. El autor manifiesta que su énfasis sobre el poder político de los intereses en la sociedad y la base conflictiva de la inflación deberían tomarse en cuenta para identificar el papel real de los bancos centrales en la fijación de la política macroeconómica: como expertos que deben asegurar el estado actual y futuro de la economía, así como también seleccionar los objetivos y los instrumentos necesarios para lograr un acuerdo sobre las metas. Lo que importa más es lo que los bancos centrales pueden hacer, y no si aparentemente están comprometidos en favor de una "tecnología" para un gobierno elegido en el mundo descrito por Barro-Gordon y Kidland-Prescott.

Además, el éxito del modelo de este trabajo en la predicción de la IBC y de la inflación lleva a dos sugerencias para estudios futuros de la política macroeconómica. En primer lugar, las economías monetarias podrían encontrar fructífero reexaminar el conflicto distributivo sobre la política monetaria, que resulta de los costos reales que la inflación y la desinflación representan para los grupos de interés. Si se trata a la inflación solamente como el resultado de los temores de un agente representativo acerca de que el gobierno pudiera producir sorpresas inflacionarias es sólo ocasionalmente apropiado en el mejor de los casos. En segundo lugar, sería útil agregar un elemento de precaución a las explicaciones institucionales de los patrones a largo plazo en materia de políticas, así como 
también recomendaciones sobre soluciones institucionales para los problemas macroeconómicos. Si bien los factores institucionales ciertamente determinan algunos resultados, en particular a corto y mediano plazo, los resultados de política consistentes en el tiempo podrían depender tanto de contextos políticos consistentes para aquellas instituciones como de las propias instituciones.

19 de septiembre de 1999.

\section{Referencias bibliográficas}

Alesina, A. y Summers L. "Central bank independence and macroeconomic performance: Some comparative evidence". Journal of Maney, Credit, and Banking, mayo, 1993.

Bade, R. y Parkin M. Central bank laws and monetary policy. University of Western Ontario, Mimeo, 1988.

Barro, R. y Gordon D. "Rules, discretion and reputation in a model of monetary policy". Journal of Monetary Economics 12, 1983, pp. 101-122.

Buchanan, J. y Tullock G. The calculus of consent. Ann Arbor: University of Michigan Press, 1962.

Cukierman, A. Central bank strategy, credibility, and independence: Theory and evidence. Cambridge, MA: MIT Press, 1992.

Cukierman, A., S. B. Webb y Neyapti B. "Measuring the independence of central banks and its effect on policy outcomes". World Bank Economic Review, Septiembre, 1992.

Debelle, G. y Fischer S. How independent should a central bank be? Massachusetts Institute of Technology. Mimeo, marzo, 1994.

Dombusch, R. y Fischer S. “Moderate inflation”. World Bank Economic Review, marzo, 1993.

Epstein, G. y Schor J. “Corporate profitability as a determinant of restrictive monetary policy: Estimates for the postwar United States". In The political economy of American monetary policy, T. Mayer (ed.). Cambridge, U.K.: Cambridge University Press, 1990, pp. 51-63.

Fischer, S. Adapting to inflation in the United States economy. In Inflation: Causes and effects, R. E. Hall (ed). Chicago: University of Chicago, 1982, pp. 169-188.

Goodhart, C. "Game theory for central bankers: A report to the Governor of the Bank of England". Journal of Economic Literature, marzo, 1994.

Greenhouse, S. "Showdown: The populist versus the Fed". New York Times, octubre 12, 1993, pp. D1 y D6.

Grilli, V., Masciandaro D. y Tabellini G. "Political and monetary institutions and public financial policies in the industrial countries". Economic Policy, octubre, 1991.

Kydland, F. E. y Prescott E. C. "Rules rather than discretion: The inconsistency of optimal plans". Journal of Political Economy 85, 1977, pp. 473-492.

Lohmann, S. "Optimal commitment in monetary policy: Credibility versus flexibility". American Economic Review, marzo, 1992.

Mc.Callum, B. “Two fallacies concerning central bank independence”. American Economic Review, mayo, 1995. 
Olson, M. The logic of collective action. Cambridge, MA: Harvard University Press, 1965.

Posen, A. "Why, central bank independence does nor cause low inflation: There is no institutional fix for politics". In Finance and the international economy: 7, R. O'Brien (ed.). Oxford: Oxford University Press, 1993, pp. 40-65.

Posen, A. "Central bank independence and disinflationary credibility: A missing link?" Federal Reserve Bank of New York Staff Reports 1, mayo, 1995.

Powell, G. B. Contemporary democracies: participation, stability, and violence. Cambridge, MA: Harvard University Press, 1982.

Rae, D. The political consequences of electoral laws. New Haven: Yale University Press, 1967.

Rogoff, K. "The optimal degree of commitment to an intermediate monetary target". Quarterly Journal of Economics, noviembre, 1985.

Santoni, G. J. "The effects of inflation on commercial banks". Federal Reserve Bank of St. Louis Review, marzo, 1986.

Walsh, C. "Central bank independence and the costs of disinflation in the EC". University of California, Santa Cruz, Mimeo, junio, 1994.

\section{Notas}

1. Banco de Reserva Federal de Nueva York. Este ensayo fue preparado para el simposio sobre Independencia de Bancos Centrales, Buró Nacional de Investigación Económica, Conferencia Décima Conferencia Anual sobre Macroeconomía, 10 y 11 de marzo de 1995. Los puntos de vista expresados en este documento son solamente los del autor, y no son aquellas del Banco de Reserva Federal de Nueva York ni del Sistema de Reserva Federal ni de ningún otro miembro de las mismas. El autor agradece a la institución Brookings por su apoyo financiero a través de la Subvención del Okum Memorial de 1993-1994. La asistencia excelente de investigación de Way-Ting Chen permitió la inclusión de países que no pertenecían a la OECD dentro de la muestra que presenta este ensayo. El autor también agradece las discusiones con Benjamín Fredman, Robert Glauber, Charles Goodhar y Robert Lawrence, y comentarios adicionales del NBER Anual y sus editores, Alberto Alesina, Barri Bossworth, John Flemming, Williaman Gale, Rick Mishkin, Dan Sichel, John Williamson y participantes en el seminario de la Universidad de Harvard, la Institución de Brooking y el Banco de Reserva Federal de Nueva York. Todos los errores remanentes son responsabilidad del autor. Apareció una versión muchísimo anterior de este argumento en Posen (1993).

2. Véanse Barro y Gordon (1983) y Kydland y Prescott (1977) para modelos originales del por qué los gobiernos deberían de quedarse con sus manos (inflacionarias) atadas. Rogoff (1985) presenta modelos de que por qué sería óptimo para algunos gobiernos que están enfrentando este dilema, establecer un banco central independiente, dado el conjunto particular de shocks y restricciones que enfrentan.

3. La relación entre el IBC y la inflación se demuestra, más adelante, como que se mantiene en países en desarrollo bajo ciertas circunstancias (véase la Sección 4).

4. Los ejemplos van desde Marbury vs. Madison (1803), en que la Corte aseguró sus atribuciones de revisión judicial a costa de permitirle a Madison que rechazara los federalistas nombrados para tribunales federales, hasta Webster vs. Planned 
Parenthood (1992), en que el "bloque moderado" se abstuvo de cambiar el precedente de Roe vs. Wade por mantener la legitimidad en la corte (un hecho explícito en la opinión de la mayoría).

5. Por supuesto, este proceso político en sí mismo está parcialmente definido por importantes instituciones políticas nacionales, tales como las constituciones y las estructuras partidarias. El autor diría, sin embargo, que éstas son diferentes de las instituciones políticas tanto en longevidad como en falta de respuesta a las presiones de los grupos de interés.

6. Así como un político debe buscar la reelección como una meta para lograr otras metas, los bancos centrales deben buscar la autonomía para fijar una política monetaria que les permita lograr otras metas, tales como la estabilidad de los precios en el largo plazo.

7. En este trabajo se considera que una mayor IBC es equivalente a aumentar el peso relativo de la estabilidad de precios sobre las metas de empleo en la función de utilidad de la autoridad monetaria. Este tratamiento está de acuerdo con el banco central conservador de Rogoff (1985), las medidas explícitas utilizadas por Cukierman, Webb y Neyapti (1992) y Grilli, Masciandaro y Tabellini (1991), y los diversos cambios institucionales basados en el Banco de Reserva de Nueva Zelandia. Según señala Goodhart (1994), la IBC tiene que ser vista como un fortalecimiento de las preferencias antiinflacionarias del banco central: "de otra manera habría que señalar que el ejemplo del Banco Central de Rusia, independiente del poder ejecutivo, pero sin un mandato en favor de la estabilidad de precios, haría volar en pedazos la correlación empírica entre la independencia y la baja inflación. Regresamos a este punto en la Sección 4.

8. Greenhouse (1993).

9. Rogoff (1985) también se refiere directamente a la comunidad financiera como una fuente de esta preferencia, aunque más brevemente.

10. Utilizando una medotología que no distingue entre la inflación anticipada y su variación, Epstein y Schor (1989) encuentran una relación negativa entre las utilidades bancarias y la inflación no anticipada y la anticipada en Estados Unidos para los años 1956 a 1985; desde que el nivel y la variación de la inflación tienen una correlación positiva, el resultado de Epstein y Schor es consistente con los resultados de Santoni.

11. Esto, por supuesto, se aleja del papel del banco central como cámara de compensación y prestamista de última instancia. Para los propósitos de este trabajo, se supone que el primer papel siempre se desempeña sin disputa, y el segundo supera cualquier otra preocupación cuando se le invoca: ninguno entra en las políticas monetarias diarias.

12. Véanse Rae (1967) y Powel (1982). La fraccionalización es igual a uno menos el índice de concentración de Herfindhal-Hirschman (que es tal vez más conocido).

13. La organización partidaria no es el único factor que determina la disciplina legislativa en un país, simplemente es la más estable y más fácilmente observable. Un conjunto de medidas más detalles escogería estos eventos como el mayor poder de los senadores y los representantes en el Congreso estadounidense desde las reformas de 1974 , a pesar de la continuación del sistema bipartidario.

14. Obtenido de varias fuentes; el autor tiene una lista disponible por países. 
15. La información de Regpow para los países de la OCDE está tomada de Grilli, Masciandaro y Tabellini (1991); los datos para países que no pertenecen a la OCDE se tomarớn de fuentes nacionales.

16. Tomado del Atlas Político del Mundo, distintos años.

17. Los valores para los países de la OCDE se tomaron de Grilli, Masciandaro y Tabellini (1991); los valores para los países que no pertenecen a la OCDE fueron calculados directamente del Atlos Político del Mundo, distintos años.

18. La inflación moderada se definió como promedios de 10 años de menos de 30 por ciento al año, como en Dornbusch y Fischer (1993). En aras de la brevedad, no se incluyeron las observaciones individuales de década/país. El autor tiene una lista completa de medidas disponible.

19. Los mismos diecisiete incluidos en las muestras de Grilli, Masciandaro y Tabellini (1991) y de Alesina y Summers (1993).

20. Singapur fue codificado a partir de 1970 , ya que en 1960 era miembro de la Federación Malaya.

21. Como la hiperinflación persistente induce cambios en la estructura económica de la sociedad, puede esperarse que los sectorres financieros en una economía hiperinflacionaria repetitiva se adapten a su ambiente, y que cambien su relación con la inflación en vez de oponerse a ella. En esa base Brasil y Argentina, aunque codificados, fueron excluidos de la muestra para propósitos estadísticos. La consistencia de la experiencia de estos países con el espíritu de este trabajo se analiza en la Sección 4.

22. El índice OFI desarrollado en este trabajo y sus componentes predicen significativamente las otras tres medidas de la IBC. Por ejemplo, una regresión OLS del índice de Alesina-Summers sobre la OFI y una constante para las observaciones de la muestra de la OCDE por décadas a través de 1950-1989, produce un coeficiente positivo significativo (al 0,5 por ciento) en OFI, con un $R 2$ ajustado de 0,50 . En la regresión del índice de Alesina-Summers de los componentes de la OFI utilizando las misma muestra, todos los coeficientes tienen el signo previsto, y todos excepto el coeficiente Fractn son significativos al nivel del 1 por ciento de nivel, con $R 2$ ajustado de 0,53 .

23. Ya que la participación en un régimen de tipo de cambio fijo no tiene poder predictivo para la IBC en cualquiera de las submuestras de la OCDE (véase Cuadro 1) o en una muestra más amplia que no se incluye, estas dos formas de restricción institucional sobre la política monetaria no pueden ser consideradas sustitutos. Este resultado vale la pena examinarlo un poco más, dado que implica que por lo menos uno de éstos no aumenta la credibilidad de la manera comúnmente supuesta, o que los gobiernos tienen una función utilitaria extraña en cuanto a los beneficios del régimen monetario.

24. Cukierman (1992) y Cukiernan, Webb y Neyapti (1992).

25. Por ejemplo, Cukierman (1992) atribuye la distinción OECD-no OECD en parte a "una norma de adherencia general a la ley", la cual se sostiene que está quizá más profundamente enraizada en las democracias occidentales desarrolladas que en los regímenes absolutos o las democracias más nuevas" (p. 454).

26. El autor agradece a John Williamson la sugerencia de que tomara en consideración el fenómeno de los bancos brasileños favorables de la inflación. Los detalles sobre las tácticas bancarias brasileñas descritas en este párrafo fueron tomados de Financial 
Times, 21 de marzo de 1990; LDC Debt Reports, 5 de septiembre de 1994; y Banker, enero de 1995.

27. Esto es, a menos que la unidad europea se diera por sentada. Si las reglas de la UE pasaran a ser indiscutibles, disminuiría su fragilidad a la alteración de uno de los bloques básicos y el Banco Central Europeo perdería parte de su protección suplementaria contra riesgos. Pero ésta es una preocupación a largo plazo. 